\title{
Status of the BONuS12 Radial Time Projection Chamber
}

\author{
G. Charles*, S. Bueltmann, G. Dodge, N. Dzbenski, M. Ehrhart, S. Kuhn, D. Payette, \\ J. Poudel \\ Old Dominion University, Norfolk, Virginia 23529, USA \\ E-mail: charlesg@ipno.in2p3.fr \\ C. Ayerbe Gayoso, K.A. Griffioen \\ College of William and Mary, Williamsburg, Virginia 23187-8795, USA
}

\section{P. Baron, I. Mandjavidze}

IRFU, CEA, Université Paris-Saclay, F-91191 Gif-sur-Yvette, France

\section{E. Christy, I. Hakki Albayrak, A. Nadeeshani}

Hampton University, Hampton, Virginia 23668, USA

\section{H. Fenker,}

Thomas Jefferson National Accelerator Facility, Jefferson Lab, Physics Division, Virginia 23606, USA

\author{
N. Kalantarians, J. Zhang \\ University of Virginia, Charlottesville, Virginia 22904, USA
}

\section{and the CLAS collaboration}

\begin{abstract}
Part of the experimental program in Hall B of the Jefferson Lab, Virginia, USA is dedicated to studying neutron structure functions using deep inelastic scattering on nuclei. For this purpose, the BONuS12 experiment will detect low momentum recoil protons in coincidence with scattered electrons. The protons will be detected by a second-generation Radial Time Projection Chamber (RTPC) using triple Gas Electron Multiplier foils for amplification while the scattered electrons will be detected by the CLAS12 spectrometer installed in Hall B. The following article presents the status of the BONuS12 RTPC detector that will take data within the next 2 years. The main improvements made from the previous BONuS RTPC: the new electronics and mounting process are presented. We also detail some aspects of the gas simulation.
\end{abstract}

5th International Conference on Micro-Pattern Gas Detectors (MPGD2017)

22-26 May, 2017

Philadelphia, USA

*Speaker. 


\section{The BONuS12 experiment}

Jefferson Laboratory (JLab) in Virginia, USA, is operating a continuous electron beam facility. It has recently been upgraded to deliver $12 \mathrm{GeV}$ beam to Hall D and $11 \mathrm{GeV}$ to Hall A, B and C, which are the four experimental halls of JLab.

The Barely Off shell NUcleon Structure experiment (BONuS) measured the nearly free neutron structure function for the first time [1]. Based on its success a new experiment, called BONuS12 [2], will take data in 2019 to measure the neutron structure function with better precision and covering larger angles. It will detect protons between $70 \mathrm{MeV} / \mathrm{c}$ and $150 \mathrm{MeV} / \mathrm{c}$ between $45^{\circ}$ and $170^{\circ}$ in coincidence with scattered electrons. The forward part of the CLAS12 spectrometer [3] installed in the Hall B is adapted to detect the scattered electron but the baseline central tracker has an energy threshold too high to detect the low energy protons.

In line with the previous BONuS experiment, the detector selected to replace the central tracker of CLAS12 is a cylindrical Radial Time Projection Chamber (RTPC). The readout pads are located on the outer cylinder of the detector and not on the end caps. When a particle crosses the detector, it ionizes the gas. Due to a radial electric field, the electrons created during the ionization drift towards the outer cylinder. An amplification device, consisting of three consecutive cylindrical Gas Electron Multiplier foils (GEM) [4], then multiplies the number of electrons to make the signal sufficiently large to be extracted by the data acquisition system.

Compared to a TPC, the RTPC has several advantages for us. A larger number of pads can be used, which is necessary to obtain a better space resolution and stand higher rates. The drift time of the electrons in the active region is shorter in a RTPC than in a TPC, which makes the RTPC a faster detector and the gas system simpler. These advantages can only be exploited if an adapted electronics system is used and careful attention is paid when mounting the detector. These two aspects along with a careful simulation of the drift properties of the gas are the main improvements made from the previous RTPC. They are presented respectively in the following sections after a short introduction to the BONuS12 setup.

\section{The BONuS12 setup}

The RTPC will be placed in the 5 T CLAS12 solenoid which will allow the determination of the momentum of the particle and keep the Moller electrons from the beam concentrated in a $30 \mathrm{~mm}$ radius cylinder around the beam axis. The active area of the RTPC is a $400 \mathrm{~mm}$ long cylinder, with a cathode placed at a radius of $30 \mathrm{~mm}$ and an outer radius of $70 \mathrm{~mm}$ where the first GEM foil is located. We hope to reach a dead area representing less than $3^{\circ}$ in azimuth, which is far less than for the previous RTPC mounted at JLab [5]. It is also twice as long and has a $10 \mathrm{~mm}$ larger radius to reach a better momentum resolution.

From the outer part to the inner part, the BONuS12 RTPC is composed of the following elements (Fig. 1):

- the readout board. Each pad is $4 \mathrm{~mm}$ along the axis of the cylinder (z) and covers $2^{\circ}$ in phi. There are 180 rows and 96 columns for a total of 17280 pads. Each row is shifted by $1 \mathrm{~mm}$ from the adjacent one to increase the position resolution. The board has a radius of $79 \mathrm{~mm}$ and is more than $400 \mathrm{~mm}$ long to account for the mechanical structure. 


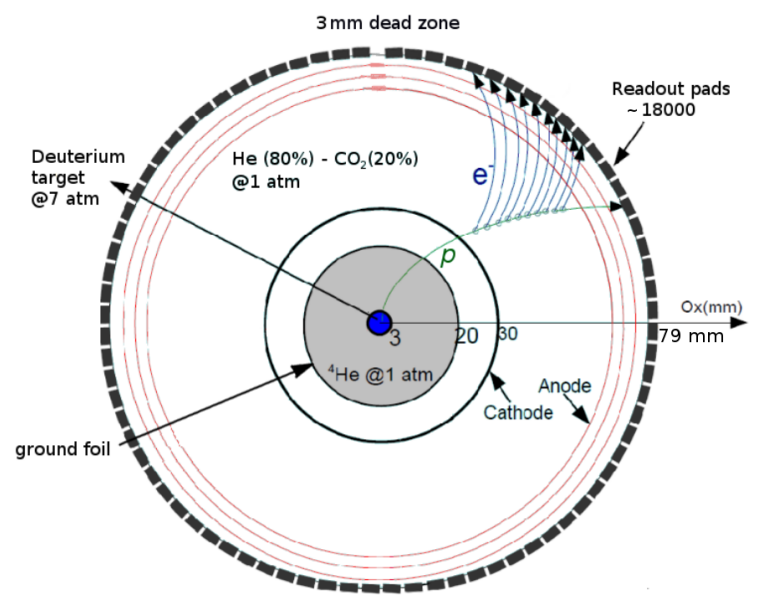

Figure 1: Schematic view of the BONuS12 RTPC.

- a cylindrical triple GEM for which the distance between foils and between the last foil and the readout pad are all $3 \mathrm{~mm}$. All the foils are $400 \mathrm{~mm}$ long and are divided on one side in 16 sectors to limit the size of a discharge.

- the cathode foil made of aluminized Mylar placed at a $30 \mathrm{~mm}$ radius.

- a ground foil made of aluminized Mylar to isolate the target from the charges on the cathode foil.

- the cylindrical target. It is a gaseous deuterium target maintained at 7 atm in a $50 \mu \mathrm{m}$ thick and $6 \mathrm{~mm}$ diameter Kapton straw.

The volume between the target and the ground foil is filled with helium while the rest of the detector is filled with a mixture of helium and carbon dioxide. This detector coupled with the detection of the electron by CLAS12 has been designed to stand a $5 \mathrm{MHz}$ particle rate and a trigger rate of $2 \mathrm{kHz}$. It is also able to separate protons from background using the energy deposition in the RTPC. The vertex resolution along the beam axis is expected to be $3 \mathrm{~mm}$ which will help to reduce the background by matching the electron and proton. Finally, the momentum resolution is expected to be $10 \%$ at $100 \mathrm{MeV} / \mathrm{c}$.

\section{Electronics}

The electronics developed for the Micromegas of CLAS12, called DREAM [6] will be connected to the RTPC. Its maximum latency is $16 \mu$ s while the maximum drift time in the RTPC is expected to be around $5 \mu$ s. The electronics has been developed for detectors with long readout elements compared to our detector and to detect minimum ionizing particles (mip). BONuS12 has small readout pads and will detect low momentum protons, that is to say particles that ionize a lot more than mip. Thanks to the possibility of changing the gain of the triple GEM as well as the dynamic range of DREAM, a good signal over noise ratio is easily achievable with these electronics for BONuS12. 
The firmware has partly been adapted to read the $5 \mu$ s and look for one or multiple signals over threshold anywhere in the $5 \mu$ s needed for the drift of the electrons in the chamber. Also, the DREAM boards of CLAS12 do not have protection from over current but a discharge can occur in the GEM foil and would damage the electronics if no protection is added. A protection board must hence be inserted between the DREAM electronics and the detector. The protection boards will be positioned around the detector as shown by fig. 2 . The main components are two diodes mounted head-to-tails to limit the amount of current in the electronics.

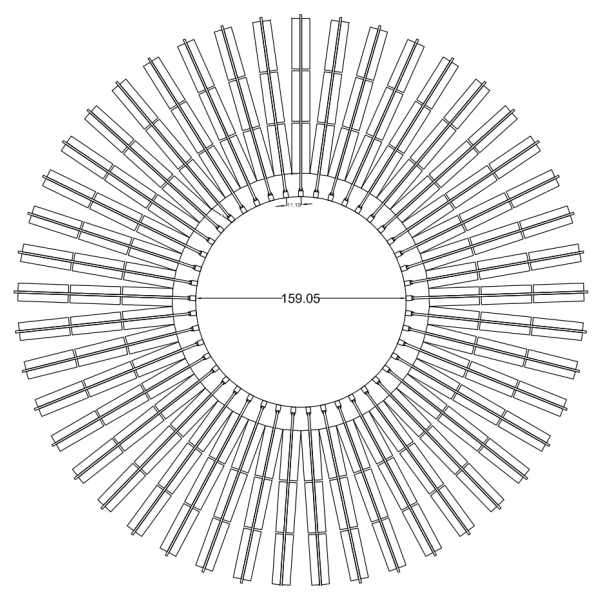

Figure 2: Schematic view of the BONuS12 RTPC with protection boards.

The DREAM electronics had never been tested with our type of detector. A test bench was mounted to ensure that a signal can be read as well as to study the length of the signal. It will be used later to check the simulations. The test bench is composed of a prototype of RTPC built for previous experiments. One of the first signals recorded with the DREAM electronics reading out the RTPC is shown fig. 3. The signal is obtained with cosmic rays in a mixture of $\mathrm{Ar} / \mathrm{CO}_{2}$ (80/20).

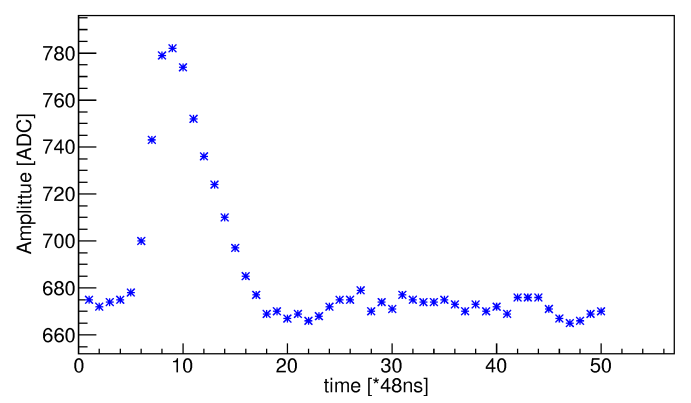

Figure 3: One signal collected by DREAM electronics when connected to a prototype of the RTPC.

First of all, this proved the ability of DREAM to read a signal from the RTPC. Then, we used these results to study the length of the signal to evaluate the expected occupancy. In this configuration, where the induction gap between the readout pads and the outer GEM foil is $4 \mathrm{~mm}$, the signal spread over about $680 \mathrm{~ns}$. It is expected to be about a fourth shorter when the induction gap will be $3 \mathrm{~mm}$. This information, coupled with the expected rate $(5 \mathrm{MHz})$ and number of pads 
hit per track (90 maximum), allowed us to determine that the occupancy will be about $1.25 \%$. The signal over noise ratio also seems comfortable. After taking more data and analyzing the pedestal, this ratio has been established to be at least 10. In this section we have demonstrated the ability of DREAM to read a RTPC and hence the BONuS12 RTPC. It also has an integration time that will keep the occupancy low enough despite the $5 \mathrm{MHz}$ particle rate.

\section{Mounting the detector}

Mounting the detector will include a multi-step process inspired by the method used to mount the RTPC like detector developed for KLOE [7]. We are particularly paying attention to the sequence in which the GEM foils will be assembled to the main support structure, the upstream end cap of the RTPC. A specific tool is being designed (fig. 4). The foil will be folded around a mandrel, then glued to form the cylinder as well as to rings at its ends. It is then slid away. The mandrel will then pivot from horizontal to vertical orientation to attach the foil to the end cap. With the current design, the first foil mounted is the inner one. The middle and outer foils are mounted successively using the same mechanism. As no bending issue is expected, no spacer will be installed between the foils.
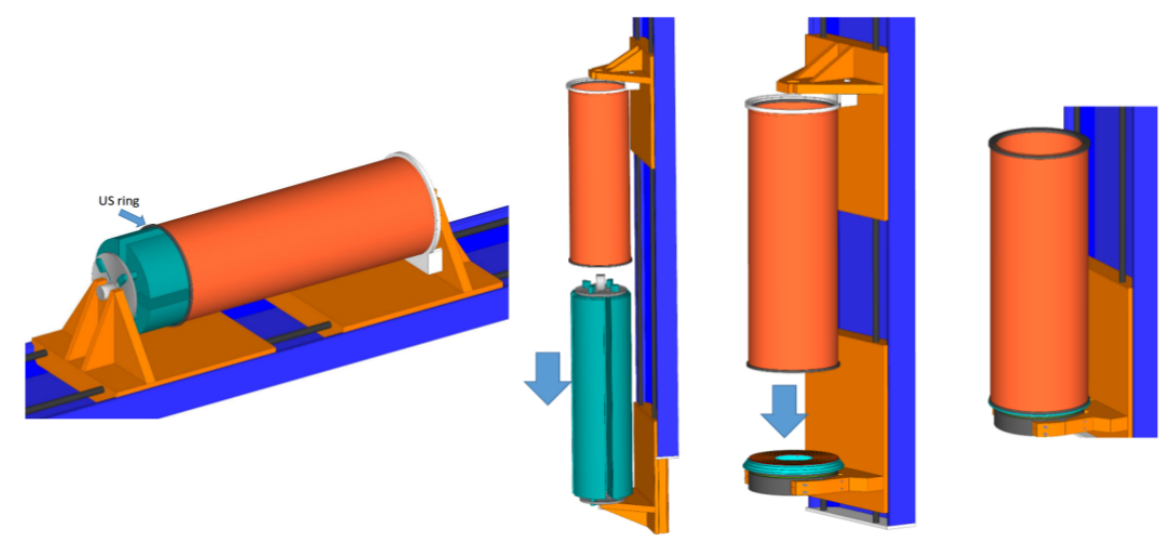

Figure 4: Scheme of the mounting process of the GEM foils on the RTPC downstream end plate.

Gluing tests have been carried out to check our ability to overlap the two sides of the same foil by only $3 \mathrm{~mm}$, which is the aimed dead area. The tests have been successful using DP 190 epoxy with 22 GA 1/2" tip.

\section{Simulation}

The first aspect of the simulation that was carried out is the study of the electron drift time and angle as a function of the gas. The previous detectors used a mixture of He/DME but for safety aspects, it is now easier to not use a flammable gas like DME. Together with the lowest drift time 
and angle, BONuS12 requires a light gas to ensure that low energy particles have a longer path. That is why mixtures with helium have been studied.

The simulation of the RTPC is based on the free software Gmsh [8] combined with Elmer [9]. The first one creates the finite element structure of the detector from the definition of the geometry. The second one evaluates the field in each element. It also produces an output that can be loaded in Garfield++ [10] to evaluate the displacement of the electrons in the detector. The drift time as a function of the drift angle for different gas mixtures as well as their variations is summarized (fig. 5).
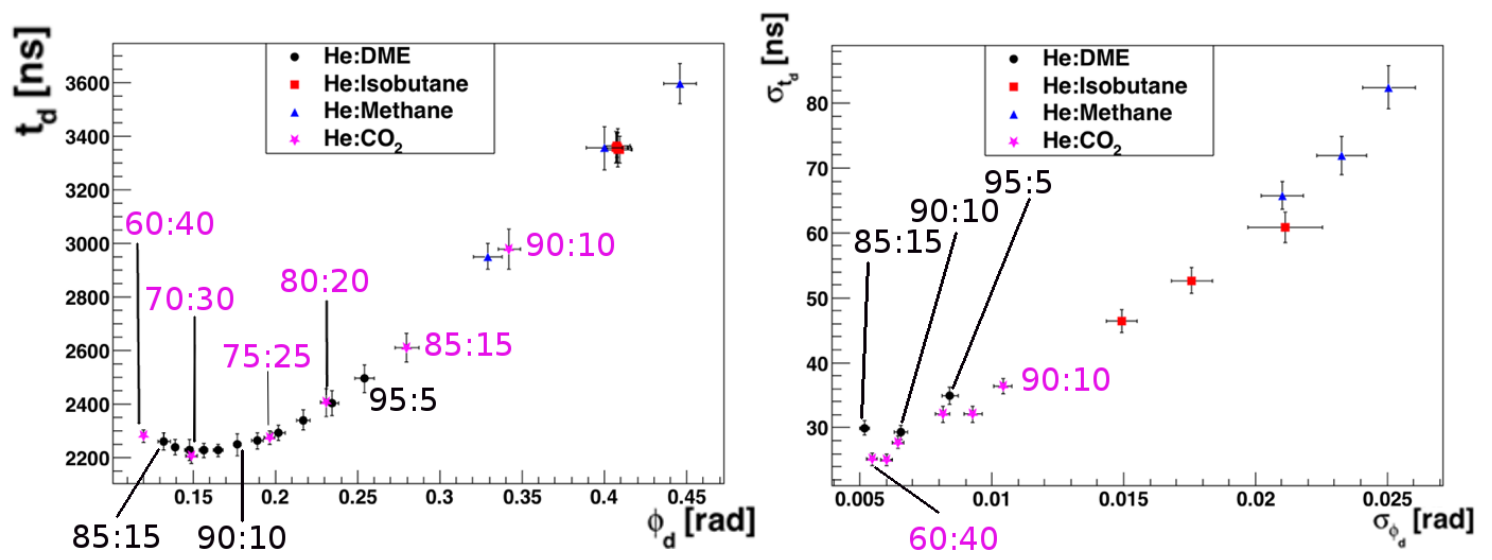

Figure 5: Drift time as a function of the drift angle for different gas mixtures (left) and its RMS variations (right). For clarity not all concentrations are indicated, the lower the helium fractions are on the left of the graphs. Helium/Methane and Helium/Isobutane mixtures where tested for 80/20, 85/15 and 90/10.

The two plots are obtained by generating electrons near the cathode foil and looking at their time and position of arrival at the inner GEM foil when the potential between the cathode and anode is $2500 \mathrm{~V}$. The conclusion from the two plots is that the best gas in our configuration is a mixture containing $70 \%$ of $\mathrm{He}$ and $30 \%$ of $\mathrm{CO}_{2}\left(\mathrm{He} / \mathrm{CO}_{2}(70 / 30)\right)$ as it has the shorter drift time and angle. The variations are also smaller for this gas. We will further study and compare the simulation with data for $\mathrm{He} / \mathrm{CO}_{2}$ (70/30) and (80/20). In particular, we will carefully check the value of the signal over noise ratio.

These results and the response of the electronics have been integrated in a simulation of the whole experiment to evaluate the position resolutions of our detector. The results are shown fig. 6 .

The radial resolution is $0.25 \mathrm{~mm}$, the azimuthal resolution $0.014 \mathrm{rad}$ and the position resolution along the beam axis is as large as the pad size in this direction. The resolutions obtained correspond to the expectations for this type of detector and to what can be expected from the previous RTPC built. Further simulations have shown that a resolution of $10 \%$ for protons of $100 \mathrm{MeV} / \mathrm{c}$ is reached. Background rejection will be studied later. A careful study of the gas mixture as hence allowed us to optimize the performances of the RTPC by reducing the drift time and drift angle. 

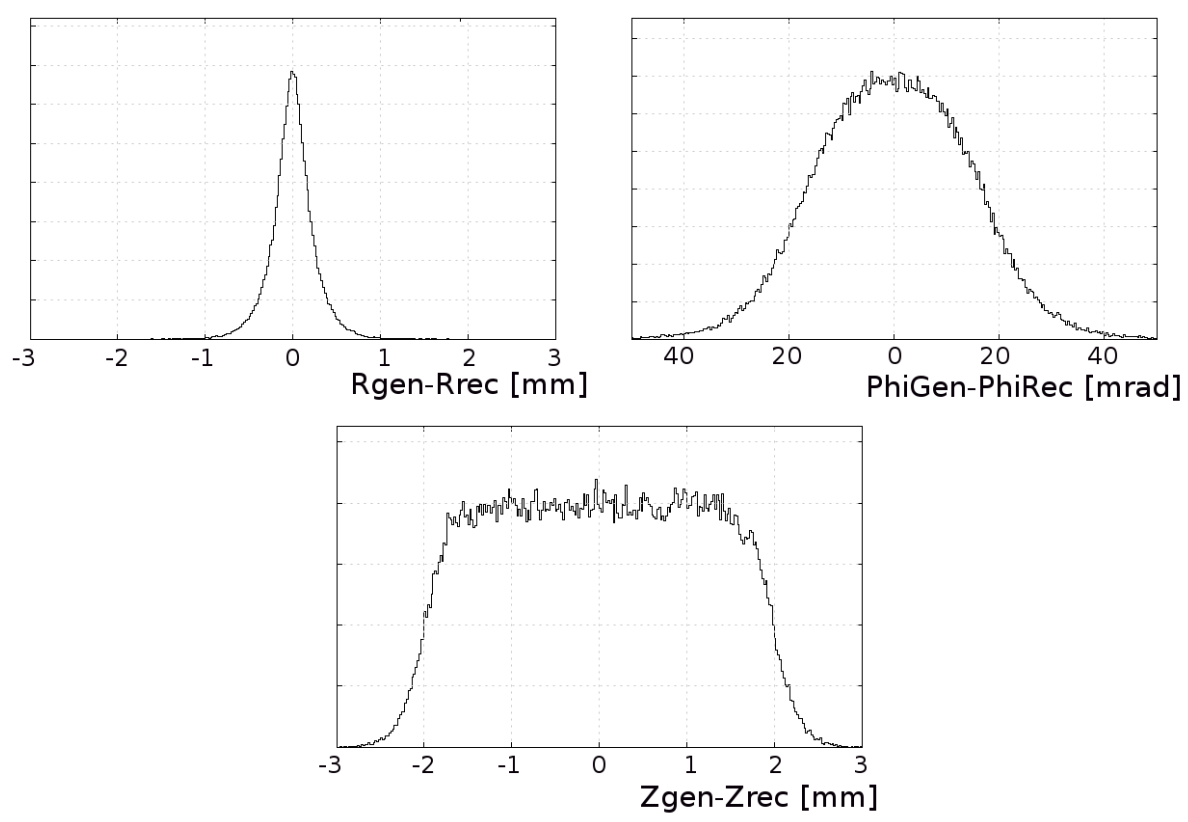

Figure 6: Radial (top left), azimuthal (top right) and z (bottom) resolution simulated for the BONuS12 RTPC.

\section{Conclusion}

BONuS12 will build a new RTPC and take data in 2019 with CLAS12 at JLab. Thanks to improvements on the design the dead area should be smaller than $3^{\circ}$ in azimuth. DREAM electronics can be used for BONuS12 and the firmware will be adapted to better fit the needs of a RTPC. The gas mixture has been studied carefully and the best non flammable mixture appears to be a mixture of $\mathrm{He} / \mathrm{CO}_{2}$ of which the exact proportion will be decided later. Finally, the position resolutions have been obtained and correspond to what one can expect from such kind of detector. The assembly of the detector is being finalized to be ready to mount a first detector by the end of 2018.

\section{Ackonowledgments}

This research was supported by the U.S. Department of Energy Office of Science under Grant No. DE-FG02-96ER40960 and by the National Science Foundation under grant No. NSF Award 1700333. We are grateful for the support from Jefferson Lab staff, including Chris Cuevas, Mark Taylor, Bob Miller, and Michael Zarecky.

\section{References}

[1] H. Fenker et al. BoNus: Development and use of a radial TPC using cylindrical GEMs, Nucl. Instr. and Meth. A 592 (2008) p. 273 [10.1016/j.nima.2008.04.047].

[2] M. Amarian et al. The structure of the free neutron at large $x$-Bjorken [https://www.jlab.org/exp_prog/proposals/06/PR12-06-113.pdf] 
[3] https://www.jlab.org/Hall-B/clas12-web/

[4] F. Sauli GEM: A new concept for electron amplification in gas detectors Nucl. Instr. and Meth. A 386 (1997) p. 531 [10.1016/S0168-9002 (96)01172-2]

[5] R. Dupré et al. A Radial Time Projection Chamber for alpha detection in CLAS at JLab [https://arxiv.org/pdf/1706.10160.pdf]

[6] Flouzat, C. et al. Dream: a 64-channel Front-end Chip with Analogue Trigger Latency Buffer for the Micromegas Tracker of the CLAS12 Experiment. Proc. of TWEPP conference (2014)

[7] A. Balla et al. Status of the cylindrical-GEM project for the KLOE-2 inner tracker Nucl. Instr. and Meth. A 628 (2011) p. 194

[8] http://gmsh.info/

[9] https://www.csc.fi/web/elmer/elmer

[10] http://garfieldpp.web.cern.ch/garfieldpp/ 